\title{
La Difusión Selectiva de la Información (DSI) en el entorno empresarial a través del Documento Electrónico : una aproximación
}

\author{
Yolanda Gonzalo Balmisa \\ Damián Martínez Ferreras \\ Elba Dengler Gómez \\ Andersen Consulting. Madrid \\ Centro de Documentación
}

\subsection{Resumen}

El objetivo del presente análisis es describir la creciente importancia que ejerce el documento electrónico como canal de comunicación interna en el entorno empresarial, canal de comunicación informativa y documental, a la vez que como vehículo telemático de una cada vez más especializada Difusión Selectiva de la Información (DSI), cuyo principal gestor es el documentalista, que se erige como figura creativa y parte esencial, integrante y protagonista, de los futuros centros de excelencia informativa y documental. (Autor)

Palabras clave: Documento Electrónico. Difusión Selectiva de la Información. Entorno empresarial.

\subsection{Abstract}

The target of this paper is to describe the rising relevance that electronic document is pushing on business environment, as communication and information channel and at the same time, as telematic channel that step-by-step makes more specialized Selective Information Delivery-SID. Information Professional plays the main role in this context : creative, integrated and principal actor of the future excellent centers focus on information and documentation delivery. (Author).

Keywords : Electronic Document. Selective Information Delivery. Business Environment.

\section{Introducción}

La sociedad cimenta su potencial en la comunicación, potencial que ante el

Scire. $3: 1$ (ene.-jun. 1997) 127-138 
empuje de las nuevas tecnologías de la información (groupware, data warehouse, entorno multimedia, entorno Web, etc.) se proyecta a través de nuevas vías de comunicación, vías como el documento electrónico, protagonista indiscutible del presente Congreso.

La revolución telemática está generando una nueva estructura de los valores más universales. Uno de ellos, y el que más nos interesa en nuestro contexto, el valor de la información, está siendo dimensionado a través de canales de difusión y distribución que han trastocado las tradicionales vías de acceso al usuario: el papel, como vehículo y soporte, y la relación directa con el propio usuario.

Esta revolución puede jugar un papel decisivo, y de hecho ya lo está jugando, para facilitar esa comunicación, ese contacto entre el usuario de la información y el productor de la misma.

\section{Conceptos y objetivos}

\section{1. ¿Qué es la DSI Telemática?}

El documento electrónico es un vehículo, una herramienta sustancial para la difusión de la información en un entorno intensivo de necesidades informativas. Difundir información, interpretada previamente por el documentalista, a colectivos especializados en áreas temáticas concretas es el objetivo de lo que hemos denominado Difusión Selectiva de la Información-DSI Telemática, vía documento electrónico.

En el entorno empresarial, estos nuevos canales de DSI, transportados por el documento electrónico, potencian el papel de los documentalistas hacia una especialización y diversificación que los eleva por encima de un mero transmisor o intermediario del mensaje informativo, es decir, nace el concepto del documentalista creativo, que ante entornos con necesidades intensivas, e incluso crispadas, de información, traspasa la frontera que le separa de la mera difusión a la elaboración a posteriori de documentación terciaria.

Hablamos de DSI Telemática, tele-direccionada y tele-dirigida, hecha a la medida de colectivos especializados, componentes e integrantes de organizaciones, tales como empresas, entidades financieras, medios de comunicación, etc.

¿Qué tipología de información es susceptible de ser DSI Telemática ?. No estamos descubriendo la pólvora : el canal telemático por el que corre el documento electrónico vincula al documentalista con el usuario en dos niveles : de manera tradicional, es decir, enviando los clásicos boletines de nuevas adquisiciones, alertas bibliográficas, etc. y otros documentos de idéntica índole, pero también de manera experta, esto es, transmitiendo una información elaborada, 
creada y asimismo compleja, para un colectivo experto en áreas temáticas con un nivel muy específico de exigencias informativas.

Esta tipología informativa parte de un mismo lugar: el Centro de Documentación, productor y garante de la transmisión de la información, validada por los diferentes niveles de utilidad, en función de la tipología de los valores del documento electrónico.

\subsection{Valores del documento electrónico}

A modo de breve recapitulación, podemos establecer las siguientes características:

2.2. 1. Permite el acceso a la información de manera directa, sobre todo rápida.

Más aún, permite un acceso globalizado, tanto a un único usuario como a un colectivo numeroso.

\subsubsection{Flujo extra-vertido}

El documentalista proyecta la información a un universo de usuarios anónimos, con la esperanza de generar un ciclo de información que no se pierda en una única dirección, sino que revierta al documentalista (también mediante otro documento electrónico de petición-ampliación-elaboración de la información y la documentación), produciendo una identificación del usuario, que de esta manera abandona el anonimato para entrar en una relación directa con el documentalista.

\subsubsection{Flujo intra-vertido}

El documentalista busca y recupera la información partiendo de la demanda realizada por el propio usuario. Este flujo hacia dentro se produce a dos niveles : de una parte, el documento electrónico que solicita la información al documentalista ; de otra, el documento electrónico que obtiene, elabora y difunde el documentalista.

\subsection{4. ¿Qué podemos y que no podemos enviar como documento electrónico?}

No toda la tipología documental es susceptible de ser transmitida por el vehículo, esto es, por el documento electrónico.

En primer lugar, la ausencia de una apropiada infraestructura tecnológica puede hacer decidir y/o deshechar qué información documental será susceptible de ser transmitida ; y en segundo lugar, no toda la información y documentación conlleva en sí misma la necesidad de ser transmitida por vía telemática.

Scire. 3 : 1 (ene.-jun. 1997) 127-138 
Ante el desequilibrio del binomio infraestructura tecnológica-DSI telemática. ¿Cuál es la necesidad que se ejerce sobre la información, y al mismo tiempo la que la propia información ejerce para ser transmitida?

\subsubsection{Categorización del Universo de usuarios-agentes}

Un estudio de las peticiones que recibe el Centro de Documentación en su actividad diaria nos ha permitido establecer diferentes categorías de usuarios que responden a necesidades también muy diferentes:

- Usuario Pasivo Pasivo : este tipo de usuario no está realmente interesado en la información y, en consecuencia, no da uso a la información que recibe. Estamos hablando de un usuario que ni siquiera consulta la información. En este grupo se encuadraría gran parte del personal de administración o aquel usuario que no dispone de la herramienta informática necesaria acceder a la información.

- Usuario Pasivo : en esta categoría se encuentra un tipo de usuario que consulta la información, bien para seleccionar aquel documento que más le interesa o simplemente por estar al tanto de la actualidad informativa que genera el Centro de Documentación. En cualquier caso, es un usuario que no solicita información, sólo la consulta.

- Usuario Activo : es el que recibe la información y la asimila, pero sus necesidades de información se ven cumplimentadas con este primer paso telemático. Por esta razón, además de la consulta, el usuario realiza la petición del documento o documentos.

- Usuario Activo Activo : es aquel usuario que no sólo solicita la información que le ha sido proporcionada sino que amplía en grado sumo la consulta, convirtiéndola en una verdadera búsqueda de información, que incluso en ocasiones es necesario re-elaborar. Este tipo de usuario rompe la barrera de la distancia telemática (aunque ésta sea de un metro) y a acude personalmente al Centro de Documentación.

\section{Metodología}

Como método de apoyo para nuestro ensayo, se ha elaborado la representación gráfica de la teoría del universo de usuarios a partir de un muestreo generado por la observación de dos variables cronológicas. Según el grado de utilización del documento electrónico que nos proporciona el registro de actividad de usuarios de la base de datos de resúmenes de prensa y revistas especializadas, hemos considerado estas dos variables : variable A (sobre un único día de uso) y variable B (sobre tres meses de uso). 
El análisis estadístico de usuarios susceptibles de solicitar información tipo vía documento electrónico (contamos en este estudio con un margen de error que ronda el $12,5 \%$, en base a la desviación que representa un grupo de usuarios que en general leen por afición, más que por necesidad de información para su actividad laboral diaria), demuestra que no es oro todo lo que reluce : el éxito en la difusión de la información depende también de la respuesta del usuario ante la información disponible vía telemática.

La primera reflexión a los datos que aporta la Fig. $n^{\circ} 1$, tomado para un día de actividad, ante un universo global de 1.418 usuarios susceptibles de recurrir al documento electrónico como vía de acceso a la información (hemos de tener en cuenta que existe otro colectivo de usuarios de 301 personas dedicadas a tareas de administración y soporte que no se consideran necesitados de información por cuanto que no forman parte directamente de la actividad empresarial), la respuesta de aquéllos no parece en principio satisfactoria como indicador de una alta

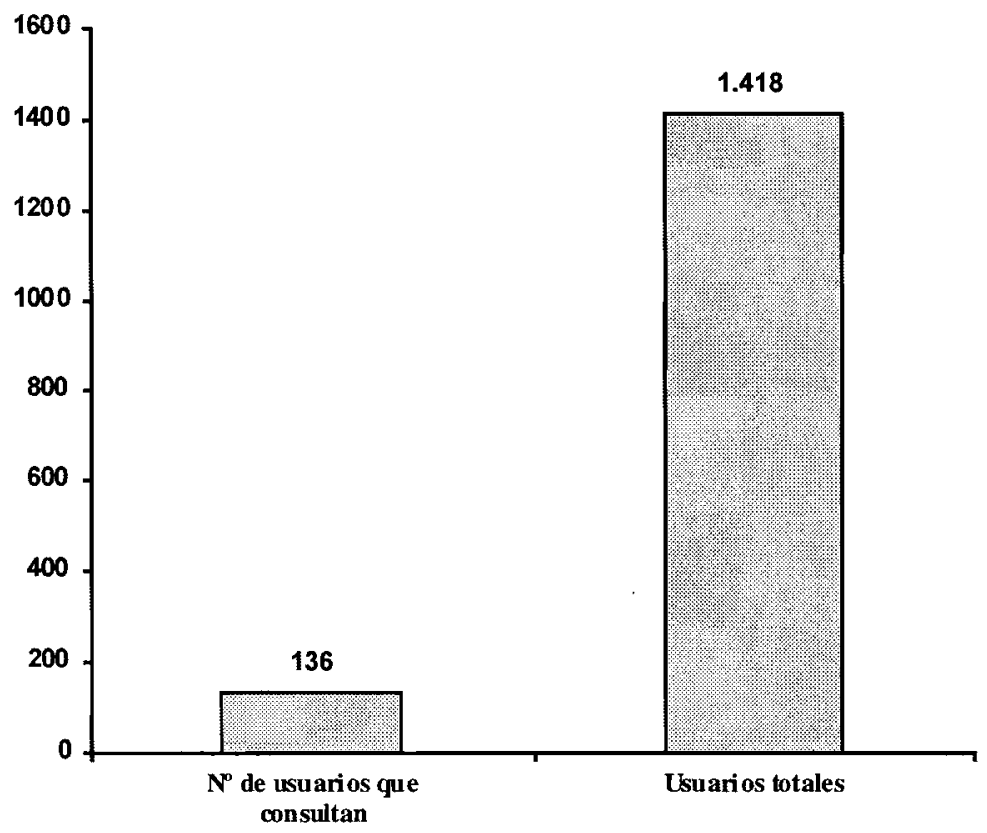

Fig. I. Grado de respuesta del usuario ante la información telem $\neq$ tica

(Variable A: I Día)

Scire. $3: 1$ (ene.-jun. 1997) 127-138 


\section{Consultas realizadas en un día (\%)}

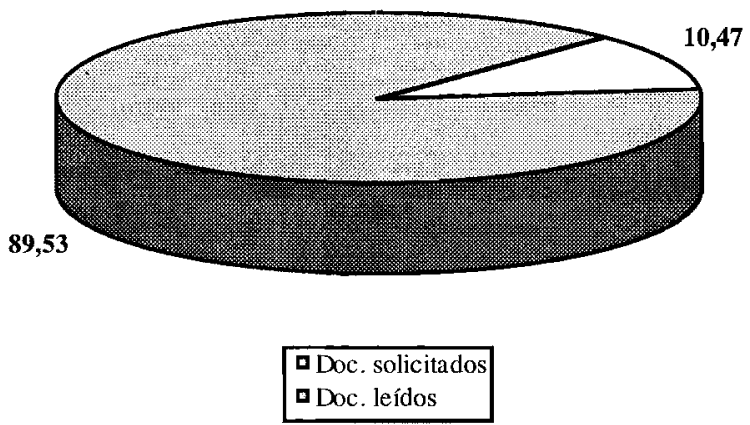

Fig. 2

frecuencia de acceso a dicha información, ya que sólo son 168 las personas que utilizan esta herramienta de información en un sólo día $(9,59 \%)$.

Sin embargo, esta cifra sí resultaría importante si verificáramos que no todos los usuarios tienen necesidad, a nivel individual, de diferente tipo de información, o que es un grupo concreto de usuarios el que consulta la información telemática para otro grupo de usuarios (vgr. secretarias para sus directores o los empleados para sus jefes). Es lo que podríamos considerar "intermediarios de la información".

Extrapolados los usuarios que hacen uso del documento electrónico se observa, tal y como se refleja en la Fig. 2, una clara diferencia entre un tipo de usuarios que únicamente consultan la información sin hacer uso explícito de ella, es decir, que no solicitan ninguna información adicional puesto que no van a hacer uso de ella (usuario pasivo-documento leído), y otro tipo de usuarios que sí están interesados en ampliar esa información concreta que reciben en formato electrónico, y recurren a la vía telemática de nuevo, es decir, mediante otro documento electrónico, que podríamos denominar formulario de petición electrónica, con lo que el usuario se convierte en usuario activo-documento solicitado. De esta manera se cierra el canal emisión-recepción-petición de información basado en el documento electrónico.

Un análisis del número de usuarios que consulta la información telemática con respecto al total de usuarios potenciales realizado durante los tres últimos meses, muestra una solución de continuidad con respecto a la tónica reflejada en 
la Fig. 1.

Así, el porcentaje de usuarios que consultan información durante este período, frente al total de usuarios potenciales, alcanza el 9,04\%, cifra ligeramente inferior al 9,56\% registrado para un sólo día pero en cierto modo comprensible si consideramos el descenso de actividad con motivo de las vacaciones navideñas.

En cualquier es digno de mención, como aspecto a resaltar, el gran volumen de documentos consultados a cargo de un número aparentemente poco representativo de usuarios, pero son éstos los denominados intermediarios de la información, los encargados de incrementar el volumen de documentos consultados.

En lo que se refiere al porcentaje de usuarios que piden información (documentos solicitados) frente a los que únicamente la consultan (documentos leídos), se mantiene, para el período comprendido entre noviembre del 96 y febrero del 97, una clara diferencia entre ambos (un $84,81 \%$ de documentos leídos frente al $15,19 \%$ de documentos solicitados), con un aumento cercano al $5 \%$ de documentos solicitados con respecto a un día de actividad.

Un análisis pormenorizado por día, semana, mes y tres meses, muestra lo que hemos comentado hasta ahora. El colectivo de usuarios que utiliza el documento electrónico como vehículo de información (168 sobre un total de 1.418) es el

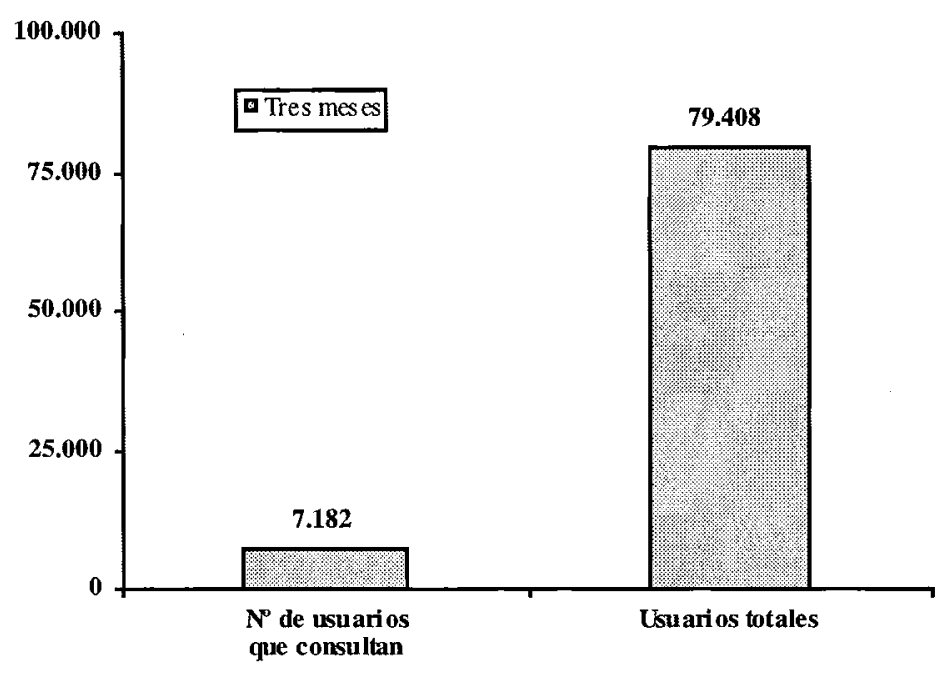

Fig. 3. Grado de respuesta del usuario ante la información telemática (Variable B: 3 Meses) 


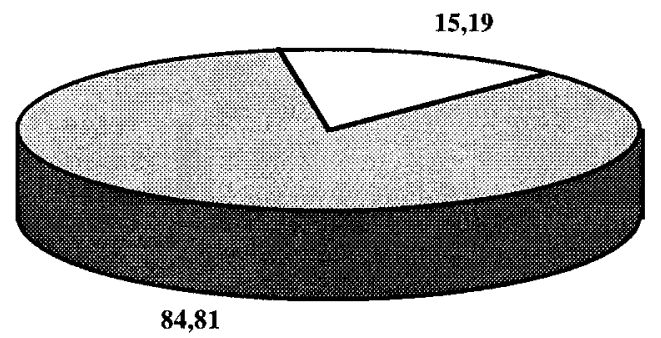

a Doc. leídos

Doc. solicitados

Fig. 4

encargado de canalizar la información para otro colectivo aún mayor y es este colectivo el que vamos a analizar a continuación, en la Fig. 5. y Fig. 6.

En el conjunto de usuarios que responden de manera efectiva al envío de la información telemática, es decir, que responden al documento electrónico, la mayor parte de ellos consultan esa información; son los denominados usuarios pasivos que simplemente quieren mantenerse informados.

Una cifra sensiblemente inferior también consulta la información disponible telemáticamente, pero, además, solicitan esa información (usuarios activos), activando el ciclo informativo mediante la solicitud del documento primario.

Además, podemos diferenciar entre estos últimos, los que simplemente solicitan la información facilitada por el Centro de Documentación y los que se interesan por obtener información complementaria, (usuario activo-activo) que necesita dar valor añadido a la información que, vía telemática, ha obtenido en un principio.

Según se desprende de la Fig. 6, y reflejado de una manera mucho más nítida que la expresada por los puros datos numéricos de la Fig. 5, la drástica separación entre la línea que representa la actividad de consulta (el sumatorio de documentos leídos más el de los documentos solicitados) entre el volumen de documentos leídos por vía telemática, frente al volumen de documentos solicitados mediante el mismo procedimiento, marca un notable despegue de los primeros frente a los 


\begin{tabular}{|l|l|l|l|}
\cline { 2 - 4 } \multicolumn{1}{c|}{} & $\begin{array}{c}\text { Número de } \\
\text { consultas }\end{array}$ & $\begin{array}{c}\text { Documentos } \\
\text { Leídos }\end{array}$ & $\begin{array}{c}\text { Documentos } \\
\text { solicitados }\end{array}$ \\
\hline Un día & 1.604 & 1.436 & 168 \\
\hline Una semana & 11.206 & 9.671 & 1.535 \\
\hline Un mes & 39.780 & 33.846 & 5.934 \\
\hline Tres meses & 111.018 & 94.145 & 16.873 \\
\hline
\end{tabular}

Fig. 5. Número de consultas realizadas vía documento electrónico (noviembre 96-febrero 97)

segundos.

Esta actividad demuestra no sólo la teoría del uso de la documentación telemática, sino su conformidad como método informativo congruente, pues el usuario se siente satisfecho con la información facilitada a través de los resúmenes de los documentos y no siente la necesidad de solicitar el documento original.

La Fig. 7 marca en definitiva, entresacados los documentos como mera actividad de consulta informativa, la espectacular diferencia que intentábamos expresar en las figuras anteriores (Fig. 5 y Fig. 6).

\section{Conclusiones. Ventajas e inconvenientes de la DSI telemática}

\subsection{Democratización informativa}

El acceso a la información vía documento electrónico se democratiza, llegando a unos niveles de difusión que difícilmente serían alcanzados de manera tradicional. Otro punto de vital importancia, y en estrecha relación con el proceso democratizador, es la eliminación progresiva de las parcelas de poder informativo que se generan en las organizaciones. La posesión de la información como arma estratégica se difumina hasta desaparecer : la información llega a todos, y son los usuarios los que deciden, como se ha demostrado, hacer o no uso de ella.

\subsection{Efectos de cambio en los documentalistas y profesionales de la infor- mación}

El documento electrónico tiene fuertes efectos de cambio en la profesión. Obliga al documentalista a un primer esfuerzo, muy importante, de selección de la información que tradicionalmente recopila. En segundo lugar, genera un elen- 


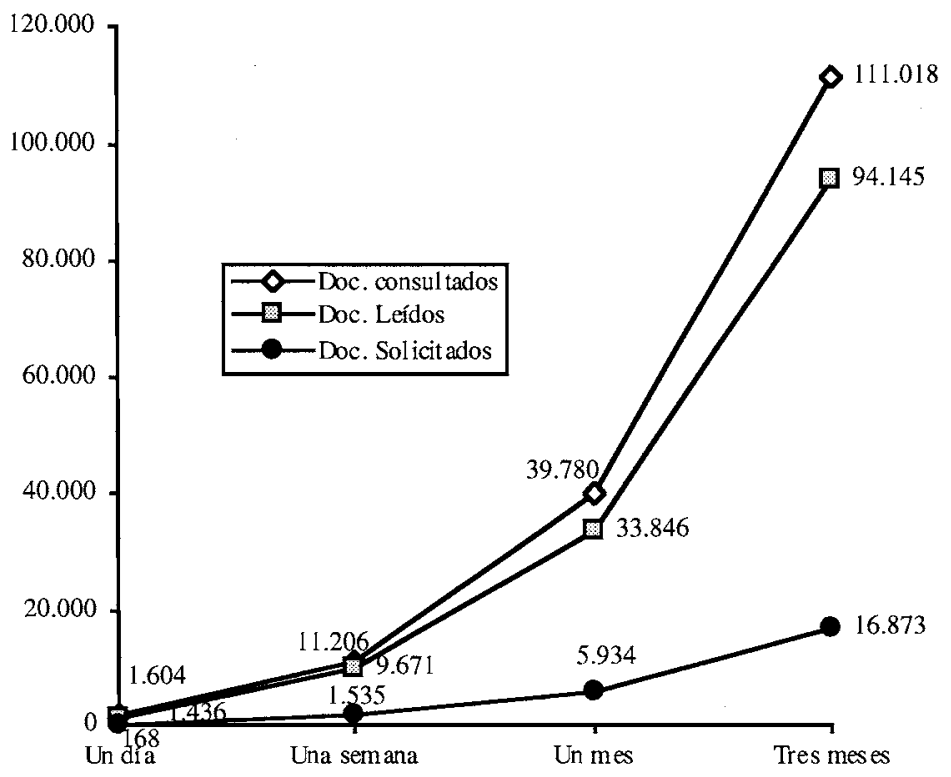

Fig. 6. Tipología de consultas realizadas vía documento electrónico

co de documentación terciaria cuyo valor expreso radica en la producción propia de esta documentación. En definitiva el documentalista deja de ser un mero transmisor, para pasar a ser a ser sujeto elaborador activo, lo que es realmente el valor intrínseco del DE.

Valorar los criterios de efectividad y alcance de la información ante un universo de usuarios dado, es decir, valorar el impacto de la misma, estructurándola y dosificándola por sectores de interés, constituye un esfuerzo creativo que ahonda en beneficio del profesional, al ser valorado como un especialista, y no como se le ha considerado tradicionalmente, como un mero agente administrativo.

La información especializada y sectorial que puede ser transmitida a través de la DSI telemática modifica sustancialmente la relación entre el usuario y el documentalista.

\subsection{Desaparición progresiva de la comunicación interpersonal}

Uno de los mayores peligros de la DSI vía documento electrónico es la progresiva desaparición de la relación directa con el usuario, relación mucho más 


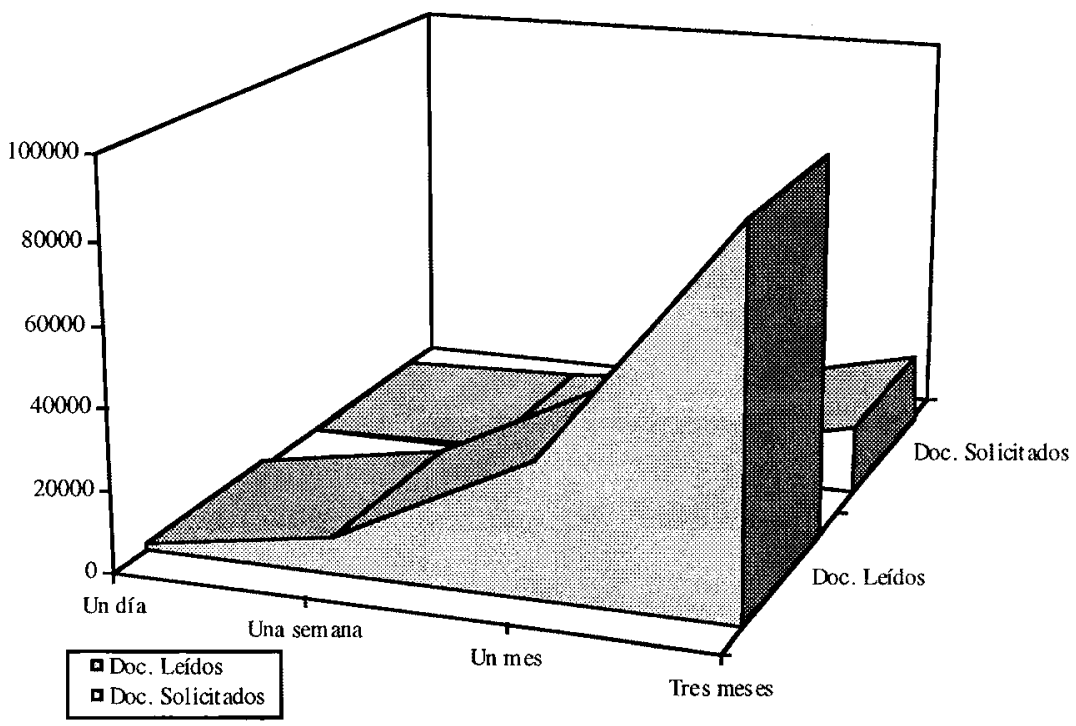

Fig. 7. Tipología de documentos electrónicos consultados (noviembre 96-febrero 97)

efectiva cuando se realiza mediante la comunicación interpersonal. El diálogo con el usuario de forma natural consigue una mayor efectividad en la búsqueda y consecución de la información. Ante el ciclo mudo que genera el canal telemático, el usuario anónimo representa un peligro que habría que tratar con suma delicadeza de cara a su implantación genérica y generalizada.

\section{Referencias}

Actitud y comportamiento de las grandes empresas españolas ante la innovación (1996).

Madrid : Círculo de Empresarios, 1996.

Buckner, Barbra ; Bowdoin, Sally (1995). Access versus Assets : A Comprehensive Guide to Resource Sharing for Academic Librarians, 1995.

Cornella, Alfons (1994). The Role of Information Services in an Information-Intensive Environment. // The Information Cycle: Production, Provision and Use (Barcelona 28-30 de Septiembre 1994). Maastricht : EADI, 1994.

Cornella, Alfons (1996). La documentación digital en al empresa. Barcelona : Marcombo, 1996.

Electronic Document Systems : User Evaluations (1993). Amazon, 1993.

Scire. $3: 1$ (ene.-jun. 1997) 127-138 
138 Yolanda Gonzalo Balmisa, Damián Martínez Ferreras y Elba Dengler Gómez

Kaser, Dick (1995). Document Delivery in an Electronic Age : A collection of Views . National Federation of Abstracting, 1995.

Mitchell, Eleanor and Walters, Sheila A (1996). Document Delivery Services : Issues and Answers. Amazon, 1996.

El Nuevo orden tecnológico (1992). Madrid : Ciencias de la Dirección, 1992

Zaid, Gabriel (1996). Los demasiados libros. Barcelona : Anagrama, 1996

Scire. $3: 1$ (ene.-jun. 1997) 127-138 